\title{
Recherches sur Setaria labiato-papillosa (Perroncito, 1882) en Afrique Occidentale.
}

\section{1 - Vecteurs naturels et vecteur expérimental,} étude des variations de la microfilarémie, infestations d'hôtes anormaux

\author{
par J. BRENGUES *, R. GIDEL ** et F. RODHAIN *** \\ Mission O.R.S.T.O.M. auprès de l'O.C.C.G.E., Laboratoire d'Entomologie du Centre Muraz, \\ Bobo-Dioulasso, Haute-Volta; \\ Laboratoire de Biologie du Centre Muraz, Sous-Section Zoonoses, \\ Bobo-Dioulasso, Haute-Volta ;
}

\begin{abstract}
Résumé
Après quelques rappels sommaires sur la position systématique, la morphologie, la biologie, le pouvoir pathogène et la transmission de Setaria labiato-papillosa, les auteurs exposent les différentes techniques utilisées pour la recherche des microfilaires: lieux et techniques de prélèvements, types de frottis utilisés, méthodes de coloration. A l'aide de différentes méthodes, ils étudient les variations de la microfilarémie au cours du nycthémère et constatent que cette filaire ne présente pas de périodicité. Enfin, ils donnent les premiers résultats d'infestations expérimentales d'animaux de laboratoire. Dans leur conclusion, ils font état des recherches en cours qui feront l'objet d'une seconde publication où figureront également les résultats des sondages parasitologiques.
\end{abstract}

\section{Summary}

The systematic position, morphology, biology, pathogenecity and transmission of Setaria labiato-papillosa are succinctly reported. Then, the authors relate the various technics used for detecting microfilariae: sampling methods and places, types of smears used, staining methods. With different technics, variations of the microfilaremia are studied on a 24 hours period: no periodicity was observed. First results of experimental infections with laboratory animals are given. Finally, actual and future researches on Setaria labiato-papillosa are described. The results of these will be published in another paper. 


\section{SOMMAIRE}

1. Introduction.

2. Rappels sommaires sur la position systématique, la morphologie, la biologie et le pouvoir pathogène de Setaria labiato-papillosa. Transmission naturelle.

2.1. Position systématique.

2.2. Morphologie.

2.3. Biologie.

2.4. Pouvoir pathogène.

2.5. Transmission naturelle.

3. Techniques de laboratoire utilisées pour la recherche des microfilaires.

3.1. Lieux et techniques de prélèvements.

3.2. Types de frottis utilisés.

3.3. Méthodes de coloration.

3.3.1. Frottis humides.

3.3.2. Frottis minces.

3.3.3. Gouttes épaisses calibrées.

4. Etude des variations de la microfilarémie au cours du nycthémère.

4.1. Techniques utilisées.

4.2. Résultats.

5. Infestations expérimentales d'animaux de laboratoire. Transmission expérimentale.

5.1. Techniques d'infestation expérimentale des animaux.

5.2. Résultats.

6. Conclusion.

7. Résumé.

8. Bibliographie.

\section{Introduction.}

Au cours des recherches entreprises sur la transmission des filaires par les Culicidés dans la région de Tingrela en Haute-Volta, l'un d'entre nous a observé la présence fréquente de larves infestantes de filaires autres que Wuchereria bancrofti (Cobbold, 1877) chez différentes espèces de moustiques. La détermination de ces larves, faite par le Professeur Nelson (London School of Hygiene and Tropical Medicine) a montré que nombre d'entre elles appartenaient au genre Setaria et probablement à l'espèce Setaria labiato-papillosa (Perroncito, 1882), parasite fréquent des bovidés en Afrique.

* Entomologiste Médical de l’Office de la Recherche Scientifique et Techniquz Outre-Mer, Laboratoire d'Entomologie du Centre Muraz, O.C.C.G.E., Bobo-Dioulasso, Haute-Volta.

** Docteur Vétérinaire, Chef de la Sous-Section Zoonoses du Centre Muraz, O.C.C.G.E., BoboDioulasso, Haute-Volta.

*** Docteur en Médecine, Assistant de Parasitologie, Service du Professeur Larivière, Facuité de Médecine de Paris, rue de l'Ecole-de-Médecine, Paris, France. 
Certaines des espèces vectrices de $S$. labiato-papillosa étant très abondantes et piquant fréquemment l'homme, nous avons été amenés à entreprendre une étude sur la transmission de cette filaire et sur la possibilité d'un rôle pathogène de celle-ci chez des hôtes anormaux. L'homme représente, en effët, l'un de ceux-ci et certaines populations rurales sont soumises à de nombreuses piqûres infestantes.

Dans cette première partie, après un rappel sommaire sur la position systématique, la morphologie, la biologie et le pouvoir pathogène de $S$. labiato-papillosa, nous indiquerons les vecteurs naturels de cette filaire ; nous décrirons ensuite les différentes techniques utilisées pour la recherche des microfilaires et donnerons les résultats d'une étude sur les variations de la microfilarémie que nous avons faite au cours du nycthémère. Pour terminer, nous exposerons les premiers résultats d'inoculations expérimentales à des hôtes anormaux (animaux de laboratoire).

\section{Rappels sommaires sur la position systématique, la morphologie, la biologie et le pouvoir pathogène de Setaria labiato-papillosa. Transmission naturelle.}

\subsection{Position systématique.}

Setaria labiato-papillosa (Syn. Setaria cervi) est classée dans la famille des Setariidae, sous-famille des Setariinae, et pour Chabaud et Anderson 1959, appartient au genre Setaria. Toutefois, certains auteurs, à la suite de Yeh (1959), classent cette espèce dans le genre Artionema où sont groupées des filaires de la sous-famille des Setariinae. parasites des Artiodactyles, et réservent le genre Setaria à une seule espèce, S. equina parasite des Equides.

\subsection{MORPHOLOGIE.}

A) Filaire adulte.

Elle se présente comme un nématode blanchâtre de dimensions variables selon le sexe et les individus: 4 à $6 \mathrm{~cm}$ sur 600 microns chez le mâle, et 6 à $9,5 \mathrm{~cm}$ sur 700-800 microns chez la femelle.

L'extrémité antérieure de la femelle présente un anneau péribuccal très saillant, portant des élévations dorsales et ventrales, fortement échancrées et bifides. L'extrémité postérieure des mâles est effilée et tire-bouchonnée. L'extrémité postérieure des femelles est enroulée en une spirale lâche (Euzéby, 1961).

B) Microfilaire.

Les microfilaires de Setaria labiato-papillosa sont longues de 220 à 260 microns et larges de 5 à 6 microns (Fain, 1955 et Williams, 1955). Elles sont pourvues d'une gaine qui déborde largement l'extrémité postérieure. Les noyaux somatiques sont arrondis ou ovalaires et s'arrêtent à 20-25 microns de l'extrémité postérieure. Un peu en arrière du milieu de la microfilaire, on distingue un corps interne unique occupant le $1 / 5^{\mathrm{e}}$ environ de la longueur totale de celle-ci. La cellule excrétrice et les cellules génitales sont visibles en coloration vitale (Fain, 1955). 


\subsection{Biologie.}

S. labiato-papillosa est une espèce cosmopolite qui se rencontre normalement à l'état adulte dans la cavité péritonéale de certains bovidés (bovins, buffles, céphalophe de Maxwell, céphalophe à flancs roux, guib harnaché) et cervidés.

Toutefois, des localisations aberrantes ont été signalées par plusieurs auteurs : foie, vessie, parenchyme rénal des bovins ; œil du cheval et de la chèvre (Euzéby, 1961).

Les microfilaires sont sanguicoles. Pour Fain (1955) et Williams (1955), il ne semble pas y avoir de périodicité. Toutefois, les observations de ces auteurs ne portaient pas sur un nycthémère complet. Nous avons suivi les variations de la microfilarémie au cours d'un nycthémère complet et à plusieurs reprises chez le même animal en prélevant du sang toutes les deux ou trois heures, et nous avons constaté que la microfilarémie subissait des variations, mais celles-ci ne traduisaient pas une périodicité (voir chapitre 4.2.).

\subsection{Pouvoir PATHOGÈNE.}

Chez les bovins, $S$. labiato-papillosa ne semble avoir aucune action pathogène. Toutefois certaines localisations exceptionnelles peuvent être à l'origine de troubles tels que la sétariose oculaire. Par contre, chez des hôtes anormaux (cheval, mouton, chèvre), la sétariose oculaire due à cette espèce est paradoxalement plus fréquente et aboutit en général à la cécité.

De plus, chez ces mêmes hôtes anormaux, cette espèce est tenue pour responsable, au Japon, d'une maladie saisonnière appelée paralysie lombaire ou filariose cérébrospinale (Itagaki, 1958) dont le pronostic est très grave. Cette affection serait due aux migrations erratiques de larves de Sétaires. D'après Shoho (1959), les formes immatures de Sétaires emprunteraient la voie nerveuse pour gagner la chambre antérieure de l'œil à travers la fente optique et, pour cet auteur, les troubles nerveux observés seraient dus au mode de cheminement de ces larves.

Enfin, S. labiato-papillosa étant transmise par des culicidés dont certaines espèces au moins sont très agressives vis-à-vis de l'homme (notamment Mansonia uniformis), il est certain que des larves infestantes de Sétaires sont inoculées à celui-ci. Danaraj (1956) et Buckley (1958) ont démontré que certaines filaires animales inoculées à l'homme étaient susceptibles de faire apparaître un syndrome d'éosinophilie pulmonaire tropicale. Comme le rappelait Nelson (1962), on ne peut écarter une étiologie filarienne pour certains troubles neurologiques ou pulmonaires associés à une éosinophilie chez l'homme dans les régions où la sétariose bovine est fréquente. L'ensemble des observations que nous avons rappelées sont en faveur d'une telle hypothèse.

\subsection{Transmission naturelle.}

En 1903, en Italie, Noé écrivait aue S. labiato-papillosa était transmise par les mouches piqueuses du bétail appartenant au genre Stomoxys. En fait, Williams (1955) n'observa pas, aux Etats-Unis, l'évolution des filaires chez ces insectes. Par contre, le 
rôle des moustiques dans la transmission de plusieurs espèces de filaire du genre Setaria est bien connu. Ainsi, les travaux de Itagaki et al. (1946 et 1947 in Euzéby, 1961), de Itagaki et Taniaguchi (1954), de Shoho et Nair (1960) ont montré que S. digitata des bovins évoluait chez les moustiques. Il en est de même en Russie, pour $S$. marshalli (= S. labiato-papillosa pour certains auteurs) des ovins (Kadenatsii, 1956).

En Afrique orientale, Heisch et al. (1959) et Nelson et al. (1962) ont constaté que Anopheles gambiae, Aedes aegypti et, surtout, Aedes pembaensis, étaient les vecteurs naturels de $S$. equina, parasite des équidés. Nos observations, effectuées en Afrique occidentale, dans la zone des savanes humides du Sud-Ouest de la Haute-Volta, montrent que dix espèces de moustiques transmettent naturellement les Setaria, filaires les plus fréquemment rencontrées chez les moustiques, avec Wuchereria bancrofti. Ces espèces vectrices sont: Anopheles funestus, A. gambiae, A. coustani, A. squamosus, Aedes dalzieli, A. fowleri, A. hirsutus, Culex antennatus, C. poicilipes, Mansonia uniformis. D'après les déterminations du Professeur Nelson (London School of Hygiene and Tropical Medicine) il semble que, très généralement, ces Setaria appartiennent à l'espèce $S$. labiato-papillosa dont le vecteur majeur serait $M$. uniformis, espèce particulièrement agressive vis-à-vis des gros animaux (bovins notamment). Au laboratoire, nous avons constaté, comme Nelson (1962) qu'Aedes aegypti est un bon vecteur expérimental (voir 5.1.A.).

\section{Techniques de Laboratoire utilisées pour la recherche des microfilaires.}

\subsection{LIEUX ET TECHNIQUES DE PRÉLÈVEMENTS.}

Trois lieux différents ont été essayés pour les prélèvements de sang: oreilles, face interne de la lèvre inférieure, jugulaire.

Les deux premiers prélèvements permettent d'obtenir du sang capillaire et le troisième du sang veineux. Il s'est avéré cependant à l'usage que la troisième technique présentait un certain nombre d'avantages sur les deux autres. En effet, les deux premières méthodes ne permettent pas toujours d'obtenir les faibles quantités de sang nécessaires aux examens $\left(20 \mathrm{~mm}^{3}\right)$, par suite de phénomènes de vasoconstriction; ceux-ci obligent souvent, de surcroît, l'opérateur à intervenir à nouveau après un certain temps (plusieurs minutes), lorsque la vasoconstriction a diminué d'intensité. Le prélèvement à la jugulaire a donc l'avantage d'être plus rapide et de permettre la récolte d'une quantité de sang importante.

\subsection{TYPES DE FROTTIS UTILISÉS.}

Trois types de frottis ont été utilisés :

- gouttes épaisses calibrées : les prélèvements étaient faits au moyen de pipettes de $20 \mathrm{~mm}^{3}$ (pipettes de Gower) et la goutte défibrinée extemporanément;

- frottis minces confectionnés suivant la technique classique ; 
- frottis humides épais fixés immédiatement dans l'alcool à $70^{\circ}$ chaud et conservés jusqu'à la coloration dans l'alcool à $70^{\circ}$ (procédé de Looss in Langeron, 1949 et Euzéby, 1958).

\subsection{Méthodes de Coloration.}

\subsubsection{Frottis humides.}

Les frottis réalisés selon la technique indiquée ci-dessus ont été colorés après déshémoglobinisation dans l'eau distillée, soit au Giemsa ( 3 gouttes pour $2 \mathrm{ml}$ pendant 20 minutes), soit à l'hématoxyline ferrique de Heidenhain (mordançage à l'alun de fer pendant 40 minutes à $37^{\circ}$ puis coloration à l'hématoxyline pendant 60 minutes à la température du laboratoire et différenciation à l'alun de fer, in Langeron, 1949).

Les résultats ont été mauvais en ce qui concerne la coloration à l'hématoxyline ferrique par suite de difficultés à obtenir une différenciation correcte des microfilaires après surcoloration. Quant à la coloration au Giemsa, les résultats obtenus étant meilleurs avec les gouttes épaisses qu'avec les frottis humides, nous avons abandonné cette dernière technique au profit de la première, à la fois plus rapide et plus simple.

\subsubsection{Frottis minces.}

Ils ont été colorés suivant la technique classique au May-Grünwald Giemsa (méthode panoptique in Langeron, 1949). Les résultats ont été satisfaisants, mais ils n'ont pas été supérieurs à ceux obtenus avec la goutte épaisse qui permet d'obtenir un plus grand nombre de microfilaires.

\subsubsection{Gouttes épaisses calibrées.}

Nous avons pu essayer les types de coloration suivants: hématoxyline ferrique de Heidenhain, hémalun normal et acide, glychémalun normal et acide, méthodes au Giemsa. D'autres colorations telles que le vert de méthyle-pyronine de Fulleborn (1941) n'ont pu être essayées, faute de disposer des colorants nécessaires.

\section{A) Hématoxyline ferrique de Heidenhain.}

Nous avons utilisé la technique décrite par Langeron (1949) en utilisant les temps suivants :

- Mordançage : à l'alun de fer à chaud pendant 15 minutes ou à $37^{\circ}$, de 20 minutes ̀े $2 \mathrm{~h} 30$ minutes.

- Coloration : soit à chaud pendant 15 minutes, soit à $37^{\circ}$ pendant 30 minutes, soit i froid pendant 15 à 20 heures.

- Différenciation: soit à l'alcool chlorhydrique pendant 3 minutes, soit à l'alun de fer pendant 15 à 30 minutes.

Quelle que soit la méthode utilisée, nous n'avons pu obtenir une différenciation satisfaisante des microfilaires. 
B) Hémalun normal et acide; glychémalun normal et acide de Mayer.

Nous avons utilisé les techniq̣ues décritẹs par Langeron (1949). Parmi celles-ci, nous avons retenu la coloration au glychémalun de Mayer qui nous a donné les meilleurs résultats et qui avait déjà été retenue par Golvan (1957). Nous avons suivi la méthode décrite par cet auteur avec les légères modifications suivantes:

- Déshémoglobinisation dans l'eau distillée au lieu de l'eau physiologique.

- Coloration pendant 20 heures environ à la température du laboratoire.

- Différentiation pendant 2 minutes maximum dans l'alcool chlorhydrique à $0,5 \%$.

- Enfin, nous avons suivi la recommandation de Langeron qui conseille de plonger les lames après le lavage, pendant quelques minutes, dans de l'eau bicarbonatée à $1 \%$.

Comme Golyan, nous avons constaté que les noyaux somatiques et sous-cuticulaires se colorent en noir violet, tandis que le corps interne n'est pas coloré. A la suite de cet auteur, nous pensons que cette coloration qui donne de bons résultats est facile à réaliser à condition toutefois d'accorder une attention particulière à la différenciation pour l'arrêter au moment' opportun.

C) Giemsa. Les trois variantes suivantes ont été essayées :

- celle préconisée par Golvan (1957): après déshémoglobinisation à l'eau physiologique, pendant 5 à 15 minutes, les lames sont colorées au Giemsa (5 gouttes pour $1 \mathrm{ml}$ d'eau distillée neutre) pendant 20 minutes;

- celle préconisée par Poirier et Deschiens (1951): hémolyse et début de la coloration par immersion pendant 10 minutes dans une solution de Giemsa à raison de 5 gouttes de Giemsa par $10 \mathrm{ml}$ d'eau distillée neutre, suivie d'une immersion de 15 à 20 minutes dans une solution plus concentrée de Giemsa ( 5 gouttes pour $1 \mathrm{ml}$ );

- celle que nous avons retenue, car plus économique en colorant et donnant des résultats aussi bons que les deux précédentes: on déshémoglobinise dans l'eau distillée, pendant 5 à $10 \mathrm{mn}$ suivant l'ancienneté du frottis. Toutefois, il nous paraît important de déshémoglobiniser dans les 48 heures qui suivent la confection de la goutte, les lames déshémoglobinisées et non fixées peuvent ensuite être conservées pendant plusieurs semaines avant coloration. Puis on fixe à l'alcool méthylique pur pendant 5 à 10 minutes. Après séchage à la température du laboratoire, on colore au Giemsa ( 3 gouttes pour $2 \mathrm{ml})$ pendant 30 minutes environ. Nous insistons, à la suite de nombreux auteurs, sur la nécessité de ne pas rejeter le colorant mais de le chasser sous le jet d'un courant d'eau, cette manière de procéder évitant la formation de précipités. Il faut aussi remarquer que, quel que soit le soin apporté à la coloration, on observe des inégalités dans la qualité de celle-ci d'un endroit à l'autre de la goutte épaisse. En particulier, nous avons constaté que la gaine des microfilaires situées à la périphérie de la goutte était mieux colorée. Pour la même concentration, nous avons essayé des temps différents: 10,20 , 30 , 40 , et 60 minutes. Il nous est apparu que la coloration à 30 minutes donnait les meilleurs résultats: gaine colorée en rouge vermillon pâle, cuticule en rouge plus foncé, noyaux assez gros et nettement séparés en violet sombre et le corps interne en rouge 
violacé avec souvent un piqueté rouge; les pores excréteur et anal sont caractérisés par une zone bleuâtre avec parfois un point rouge vermillon en leur centre. Les noyaux de la queue se terminent en une seule rangée qui n'atteint pas l'extrémité caudale.

\section{Etude des variations de la microfilarémie au cours du nycthémère.}

\subsection{Techniques utilisées.}

Trois techniques ont été utilisées :

A) Frottis épais calibrés.

Ces frottis ont été réalisés avec du sang veineux à l'aide d'une pipette de Duclos de $2 \mathrm{ml}$. Celle-ci avait été préalablement étalonnée avec du sang de bovin, et nous avions constaté qu'une goutte de sang représentait $58,8 \mathrm{~mm}^{3}$ (34 gouttes pour $2 \mathrm{ml}$ ), soit environ $59 \mathrm{~mm}^{3}$. Ces frottis, défibrinés au moment du prélèvement, étaient ensuite colorés au Giemsa suivant la technique indiquée pour les gouttes épaisses. La numération des microfilaires a été effectuée au microscope (grossissement $100 \mathrm{X}$ ).

B) Gouttes épaisses calibrées.

Ces gouttes ont été réalisées, soit avec du sang capillaire, soit avec du sang veineux, à l'aide de pipettes calibrées de $20 \mathrm{~mm}^{3}$ (pipette de Gower). Après avoir été défibrinées, elles ont été colorées au Giemsa, suivant la technique précédemment décrite. La numération des microfilaires a été faite comme pour les frottis épais.

\section{Tableau 1}

Etude de la périodicité du 5 au 6-12-66: nombre moyen de microfilaires par mm ${ }^{3}$, observé toutes les 2 heures (pré'èvements de $20 \mathrm{~mm}^{3}$ de sang capillaire à loreille)

\begin{tabular}{|c|c|c|c|}
\hline Heures & $\begin{array}{c}\text { NOMBRE DE } \\
\text { MICROFILAIRES PAR } \mathrm{MM}^{3}\end{array}$ & Heures & $\begin{array}{l}\text { NOMBRE DE } \\
\text { MICROFILAIRES } \\
\text { PAR } \text { MM }^{3}\end{array}$ \\
\hline 8 h $30 \ldots \ldots$ & 4,1 & 20 h 30 & 5,3 \\
\hline 10 h $30 \ldots \ldots$ & 2,5 & 22 h 30 & 5,6 \\
\hline 12 h $30 \ldots \ldots$ & 2,4 & 0 h 30 & 3,9 \\
\hline 14 h $30 \ldots \ldots$ & 1,7 & $2 \mathrm{~h} 30$ & 5,6 \\
\hline 16 h $30 \ldots \ldots$ & 2,5 & 4 h 30 & 3,8 \\
\hline 18 h $30 \ldots \ldots$ & 5,8 & $6 \mathrm{~h} 30$ & 4,7 \\
\hline
\end{tabular}




\section{C) Cellules de Nageotte.}

Nous avons utilisé des cellules de Nageotte d'un volume de $50 \mathrm{~mm}^{3}$. Le sang prélevé à la jugulaire était dilué au $1 / 20^{\circ}$ avec du liquide de Lazarus à l'aide de pipettes mélangeurs de Potain pour globules blancs. La dilution au $1 / 10^{\circ}$ avait été essayée préalablement, mais avait dû être abandonnée; par suite de la coloration trop intense, il était impossible de distinguer au microscope (grossissement $100 \mathrm{X}$ ) le réseau de la cellule. Avec la dilution au $1 / 20^{\circ}$, le réseau de la cellule est bien visible et les microfilaires sont immobiles et parfaitement identifiables.

\subsection{Résultats.}

Trois études complètes sur la périodicité ont été effectuées chez le même animal (taurin de sexe mâle âgé d'un an environ) à trois dates différentes, séparées par un intervalle de trois ou quatre semaines.

Dans le tableau 1 figurent les résultats de la première expérimentation. Les prélèvements ont été réalisés toutes les deux heures à l'oreille de l'animal et des gouttes épaissès de $20 \mathrm{~mm}^{3}$ ont été confectionnées. Le nombre de microfilaires porté dans le tableau 1 et les tableaux 2 et 3 a été ramené au $\mathrm{mm}^{3}$ afin de rendre comparable les différentes observations faites suivant les trois techniques décrites ci-dessus.

\section{Tableau 2}

Etude de la périodicité du 27 au 28-12-66: nombre moyen de microfilaires par $\mathrm{mm}^{3}$, observé toutes les 3 heures (sang veineux: gouttes épaisses de $20 \mathrm{~mm}^{3}$ et frottis épais de $59 \mathrm{~mm}^{3}$ )

\begin{tabular}{|c|c|c|c|c|c|}
\hline \multirow[b]{2}{*}{ HeuRES } & \multicolumn{2}{|c|}{$\begin{array}{l}\text { NOMBRE DE MICROFILAIRES } \\
\text { PAR } \mathrm{MM}^{3}\end{array}$} & \multirow[b]{2}{*}{ HEURES } & \multicolumn{2}{|c|}{$\begin{array}{l}\text { NOMBRE DE MICROFILAIRES } \\
\text { PAR MM }{ }^{3}\end{array}$} \\
\hline & $\begin{array}{l}\text { pipettes } \\
\text { de } 20 \mathrm{~mm}^{3}\end{array}$ & $\begin{array}{l}\text { pipettes de } \\
\text { Duclos } \\
\text { (gouttes de } \\
59 \mathrm{~mm}^{3} \text { ) }\end{array}$ & & $\begin{array}{l}\text { pipettes de } \\
20 \mathrm{~mm}^{3}\end{array}$ & $\begin{array}{l}\text { pipettes de } \\
\text { Duclos } \\
\text { (gouttes de } \\
59 \mathrm{~mm}^{3} \text { ) }\end{array}$ \\
\hline $9 \mathrm{~h} \ldots \ldots$ & 7,1 & - & $0 \mathrm{~h}$ & 5,5 & 6,2 \\
\hline $12 \mathrm{~h} \ldots \ldots$ & 6,2 & 6,1 & $3 \mathrm{~h}$ & 7,8 & 6,1 \\
\hline $15 \mathrm{~h} \ldots \ldots$ & 7,3 & 4,6 & $6 \mathrm{~h}$ & 6,8 & 5,8 \\
\hline $18 \mathrm{~h} \ldots \ldots$ & 6,0 & 6,6 & $9 \mathrm{~h}$ & 5,0 & - \\
\hline $21 \mathrm{~h} \ldots \ldots$ & 7,6 & 5,6 & & & \\
\hline
\end{tabular}

Dans le tableau 2 sont portés les résultats de la deuxième expérimentation. Les prélèvements ont été effectués toutes les trois heures à la jugulaire et, avec le sang recueilli, nous avons confectionné d'une part des gouttes épaisses de $20 \mathrm{~mm}^{3}$, et, d'autre part, des frottis épais de $59 \mathrm{~mm}^{3}$. 


\section{Tableau 3}

Etude de la périodicité du 31-1 au 1-2-67: nombre moyen de microfilaires par mm³ observé toutes les 3 heures (sang veineux: gouttes épaisses de $20 \mathrm{~mm}^{3}$ et cellules de Nageotte)

\begin{tabular}{|c|c|c|c|c|c|}
\hline \multirow{2}{*}{ Heures } & \multicolumn{2}{|c|}{$\begin{array}{c}\text { NOMBRE DE MICROFILAIRES } \\
\text { PAR } \mathrm{MM}^{3}\end{array}$} & \multirow{2}{*}{ HEURES } & \multicolumn{2}{|c|}{$\begin{array}{l}\text { NOMBRE DE MICROFILAIRES } \\
\text { PAR } \text { MM }^{3}\end{array}$} \\
\hline & $\begin{array}{l}\text { pipettes de } \\
20 \mathrm{~mm}^{3}\end{array}$ & $\begin{array}{l}\text { cellule de } \\
\text { Nageotte }\end{array}$ & & $\begin{array}{l}\text { pipettes de } \\
20 \mathrm{~mm}^{3}\end{array}$ & $\begin{array}{l}\text { cellule de } \\
\text { Nageotte }\end{array}$ \\
\hline $9 \mathrm{~h} \ldots \ldots$ & 6,5 & 一 & $0 \mathrm{~h}$ & 6,7 & 9,2 \\
\hline $12 \mathrm{~h} \ldots \ldots$ & 7,0 & 8,0 & $3 \mathrm{~h}$ & 4,0 & 5,5 \\
\hline $15 \mathrm{~h} \ldots \ldots$ & 7,3 & 9,2 & $6 \mathrm{~h}$ & 3,9 & 10,9 \\
\hline $18 \mathrm{~h} \ldots \ldots$ & 7,4 & 9,2 & $9 \mathrm{~h}$ & 2,5 & 7,6 \\
\hline $21 \mathrm{~h} \ldots \ldots$ & 7,4 & 8,3 & & & \\
\hline
\end{tabular}

Tableau 4

Variations du nombre des microfilaires sur plusieurs gouttes épaisses de $20 \mathrm{~mm}^{3}$ prélevées à la même heure

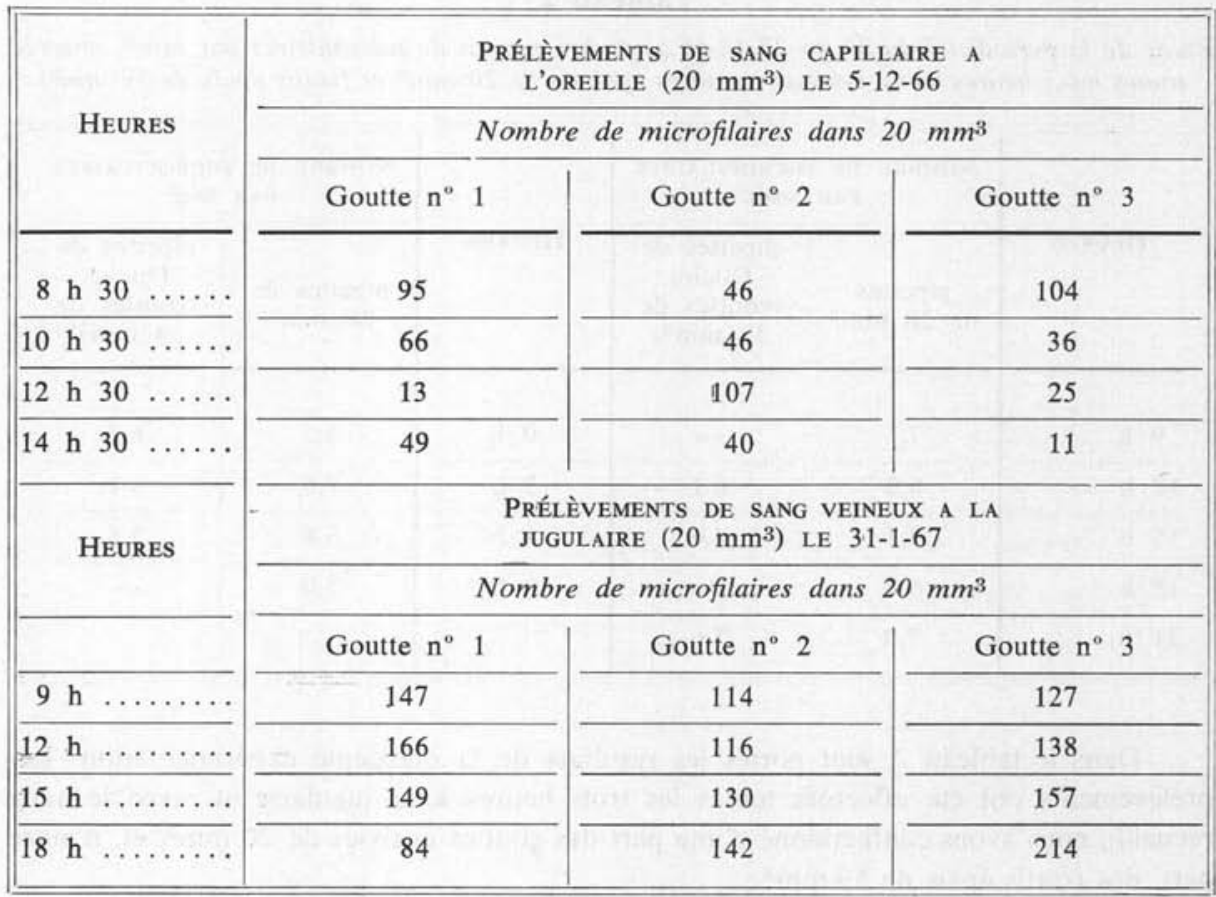


Enfin, dans le tableau 3 sont indiqués les résultats de la troisième expérimentation. Les prélèvements ont été faits toutes les trois heures également à la jugulaire. La numération des microfilaires a été effectuée soit sur gouttes épaisses de $20 \mathrm{~mm}^{3}$, soit en cellules de Nageotte.

L'ensemble des résultats portés dans les trois tableaux ne fait pas apparaître de périodicité. Certes, on constate des variations de la microfilarémie au cours du nycthémère. Cependant, celles-ci ne sont pas fonction de l'heure puisque des prélèvements pratiqués aux mêmes heures, mais à des jours différents, donnent des variations de même amplitude que celles constatées au cours du nycthémère. De même, on peut constater des variations importantes entre plusieurs gouttes confectionnées au même moment et suivant la même technique, que ce soit à partir de sang capillaire prélevé directement à l'oreille avec la pipette calibrée, ou de sang veineux recueilli préalablement dans un tube à hémolyse et utilisé immédiatement (voir tableau 4). Dans ce même tableau, on peut constater également que le nombre de microfilaires observés dans $20 \mathrm{~mm}^{3}$ est très généralement supérieur lorsqu'il s'agit de sang veineux. Cette différence est peut-être liée aux phénomènes de vasoconstriction précédemment signalée. De même, la densité microfilarienne peut varier sensiblement entre plusieurs cellules de Nageotte contënant le même sang dilué.

\section{Infestations expérimentales d'animaux de laboratoire. Transmission expé- rimentale.}

\subsection{TeChNiQues d’infestation eXPÉRIMENTALE DES ANIMAuX.}

A) Inoculation des filaires infestantes par l'intermédiaire d'un insecte vecteur.

Nous avons utilisé une souche d'Aedes aegypti L., colonisée en laboratoire depuis plusieurs années. Les femelles issues de l'élevage étaient nourries sur le veau filarien et conservées jusqu'à complète maturation des filaires. Pendant la période d'évolution des filaires, les femelles d'Aedes aegypti étaient nourries avec de l'eau glucosée à $5 \%$. Nous avons constaté, à la suite de Nelson (1962), que la durée d'évolution des filaires chez l'insecte vecteur est de 8 à 10 jours en saison sèche chaude, par contre elle peut atteindre 14 à 15 jours, et même plus, en saison froide.

Les moustiques infestants étaient gorgés sur les animaux d'expérience et le nombre eszompté de filaires libérées était estimé suivant la méthode détaillée ci-dessous (voir 5.2.).

B) Inoculation des filaires infestantes par voie intrapéritonéale.

Les femelles infestantes d'Aedes aegypti étaient disséquées dans de l'eau physiologique èt les filaires infestantes étaient recueillies et placées dans un verre de montre contenant quelques gouttes d'eau physiologique. Celles-ci étaient inoculées par voie intra-péritonéale à l'aide d'une seringue en même temps qu'une solution d'antibiotiques (pénicilline-streptomycine), afin de prévenir une infection bactérienne éventuelle. 


\section{Tableau 5}

Résultats des inoculations chez les animaux de laboratoire: i.p.: par voie intrapéritonéale, inf. nat.: infestation naturelle par l'insecte vecteur

\begin{tabular}{|c|c|c|c|c|c|c|}
\hline $\begin{array}{l}\text { ANIMAL } \\
\text { INOCULÉ }\end{array}$ & $\begin{array}{c}\text { NOMBRE } \\
\text { DE } \\
\text { FILAIRES } \\
\text { INOCULÉES }\end{array}$ & $\begin{array}{l}\text { MODE D'INO- } \\
\text { CULATION }\end{array}$ & $\begin{array}{l}\text { DATE } \\
\text { D'INOCU- } \\
\text { LATION }\end{array}$ & $\begin{array}{c}\text { DATE } \\
\text { D'AUTOP- } \\
\text { SIE }\end{array}$ & $\begin{array}{l}\text { RÉSULTATS } \\
\text { D'AUTOPSIE }\end{array}$ & $\begin{array}{l}\text { OBSERVA- } \\
\text { TIONS }\end{array}$ \\
\hline Rat $n^{\circ} 1 \ldots \ldots$ & 10 & i.p. & $15 / 2 / 67$ & $28 / 3 / 67$ & R.A.S. & - \\
\hline Rat $n^{\circ} 2 \ldots \ldots$ & 10 & i.p. & $15 / 2 / 67$ & $25 / 9 / 67$ & R.A.S. & - \\
\hline Rat $n^{\circ} 3 \ldots \ldots$ & 15 & i.p. & $18 / 2 / 67$ & $25 / 9 / 67$ & R.A.S. & - \\
\hline Rat $n^{\circ} 4 \ldots \ldots$ & 15 & i.p. & $18 / 2 / 67$ & $27 / 9 / 67$ & R.A.S. & - \\
\hline Rat $n^{\circ} 5 \ldots \ldots$ & 22 & inf. nat. & $20 / 2 / 67$ & $16 / 6 / 67$ & $\begin{array}{l}\text { lésions } \\
\text { pulm. }\end{array}$ & $\begin{array}{l}\text { troubles } \\
\text { nerveux }\end{array}$ \\
\hline Rat $n^{\circ} 6 \ldots \ldots$ & 18 & i.p. & $20 / 2 / 67$ & $29 / 9 / 67$ & R.A.S. & - \\
\hline Rat $\mathrm{n}^{\circ} 7 \ldots \ldots$ & 18 & inf. nat. & $25 / 3 / 67$ & $9 / 10 / 67$ & R.A.S. & 一 \\
\hline Rat $n^{\circ} 8 \ldots \ldots$ & 8 & inf. nat. & $25 / 3 / 67$ & $11 / 10 / 67$ & R.A.S. & - \\
\hline Rat $n^{\circ} 9 \ldots \ldots$ & 23 & inf. nat. & $28 / 3 / 67$ & $11 / 10 / 67$ & R.A.S. & - \\
\hline Rat $\mathrm{n}^{\circ} 10 \ldots \ldots$ & 11 & inf. nat. & $28 / 3 / 67$ & $11 / 10 / 67$ & R.A.S. & - \\
\hline Rat $n^{\circ} 11 \ldots \ldots$ & 12 & inf. nat. & $29 / 3 / 67$ & $8 / 1 / 68$ & $\begin{array}{l}\text { lésions } \\
\text { pulm. }\end{array}$ & 一 \\
\hline Cobaye $\mathrm{n}^{\circ} 1 \ldots$ & 10 & i.p. & $15 / 2 / 67$ & $8 / 1 / 68$ & R.A.S. & - \\
\hline Cobaye $\mathrm{n}^{\circ} 2 \ldots$ & 21 & i.p. & $18 / 2 / 67$ & $8 / 1 / 68$ & R.A.S. & 一 \\
\hline Cobaye $\mathrm{n}^{\circ} \quad 3 \ldots$ & 22 & inf. nat. & $28 / 3 / 67$ & $8 / 1 / 68$ & R.A.S. & - \\
\hline Cobaye $\mathrm{n}^{\circ} \quad 4 \quad \ldots$ & 15 & inf. nat. & $29 / 3 / 67$ & $8 / 1 / 68$ & R.A.S. & - \\
\hline Cobaye $\mathrm{n}^{\circ} 5 \ldots$ & 12 & inf. nat. & $3 / 4 / 67$ & $8 / 1 / 68$ & R.A.S. & - \\
\hline Lapin $\mathrm{n}^{\circ} 1 \ldots \ldots$ & 21 & inf. nat. & $15 / 2 / 67$ & $5 / 1 / 68$ & R.A.S. & - \\
\hline Lapin $\mathrm{n}^{\circ} 2 \ldots$ & 15 & i.p. & $15 / 2 / 67$ & $5 / 1 / 68$ & R.A.S. & - \\
\hline Lapin $\mathrm{n}^{\circ} \quad 3 \ldots \ldots$ & 15 & inf. nat. & $17 / 2 / 67$ & $1 / 12 / 67$ & - & $\begin{array}{l}\text { décédé le } \\
1 / 12 / 67\end{array}$ \\
\hline
\end{tabular}

\subsection{Résultats.}

Trois lapins, cinq cobayes et onze rats ont été inoculés. Les résultats de cette expérimentation sont portés dans le tableau 5. Le nombre de filaires infestantes inoculées a été évalué de la façon suivante : en ce qui concerne celles inoculées par voie intrapéritonéale, elles étaient comptées à la loupe stéréoscopique dans l'eau physiologique avant l'inoculation. En ce qui concerne celles inoculées par l'insecte vecteur, leur nombre était estimé comme suit : les moustiques infestants étaient divisés en deux lots ; les moustiques du premier lot étaient disséqués et le nombre de filaires infestantes était déterminé pour chacun d'eux. On pouvait ainsi établir le nombre moyen de filaires infestantes par moustique avant le repas de sang. Les moustiques du deuxième lot étaient disséqués 
après le repas de sang sur les animaux d'expérience et on pouvait ainsi établir le nombre moyen de filaires infestantes subsistant chez l'insecte après un repas de sang. A partir de ces chiffres, on pouvait évaluer le nombre moyen escompté des filaires infestantes inoculées à chaque animal. Toutefois, le nombre réel de filaires inoculées est probablement inférieur au nombre escompté par suite de pertes possibles de filaires à la surface de la peau avant leur pénétration.

Les animaux inoculés ont été sacrifiés après une période d'observation allant de un mois et demi minimum à dix mois et demi maximum ; dix-sept d'entre-eux ont été sacrifiés après six mois. Si on excepte le lapin $\mathrm{n}^{\circ} 3$ décédé accidentellement, deux animaux seulement (rats $\mathrm{n}^{\circ *} 5$ et 11 ) ont présenté des lésions à l'autopsie (pneumonie atypique). Il est toutefois nullement évident que ces lésions soient en relation avec l'inoculation des filaires. Signalons cependant que le rat $\mathrm{n}^{\circ} 5$, peu de temps avant son autopsie, avait présenté des troubles nerveux (torsion du cou, anomalie unilatérale de la démarche) pouvant évoquer la paralysie lombaire des ovins décrite en Extrême-Orient.

\section{Conclusions.}

Dans cette première partie, nous avons montré que Setaria labiato-papillosa, qui semble largement répandue en Afrique Occidentale, était transmise naturellement par plusieurs espèces de moustiques, Mansonia uniformis étant cependant le vecteur majeur dans les zones prospectées. Nous avons aussi constaté qu'une souche d'Aedes aegypti, originaire de Haute-Volta, était un bon vecteur expérimental. Les études que nous avons faites sur la variation de la microfilarémie au cours du nycthémère nous ont permis de constater qu'il n'y avait pas de périodicité. Nous avons cependant observé des variations de la microfilarémie qui sont peut-être sous la dépendance de certains facteurs tels que ceux étudiés par Hawking (1967). Nous nous proposons d'étudier ultérieurement l'influence éventuelle de ces facteurs. Les tentatives de transmission à des hôtes anormaux (animaux de laboratoire) n'ont pas été concluantes. Toutefois, du fait de la possibilité de transmission de cette filaire à l'homme, par certains des vecteurs naturels qui piquent celui-ci fréquemmént (Mansonia uniformis entre autres), nous envisageons de renouveler ces expériences en les étendant à d'autres hôtes anormaux (ovins, caprins). De même, nous tenterons l'infestation expérimentale de l'hôte normal afin de suivre l'évolution de cette filariose et les réactions éventuelles de l'hôte.

Nous étudions actuellement la fréquence et les variations saisonnières de la sétariose chez les bovins abattus à Bobo-Dioulasso et Banfora (Haute-Volta). Une étude de la dynamique de cette filariose (variations en fonction du sexe, de l'âge et de la saison) a été effectuée chez les bovins de deux troupeaux qui ont été suivis pendant une année. Enfin, des sondages parasitologiques sont prévus en plusieurs points de la zone des savanes humides du Sud-Ouest de la Haute-Volta. L'ensemble de ces résultats fera l'objet d'une publication ultérieure. 


\section{Bibliographie}

BUCKLEY (J. J. C.), 1958. - Occult Filarial Infections of Animal origin as a cause of Tropical Pulmonary Eosinophilia. E. Afr. Med. J., 35, 493-500.

Chabaud (A. G.) et Anderson (R. C.), 1959. - Nouvel essai de classification des filaires (superfamille des Filarioidea). Ann. Parasit. hum. comp., 34, (1-2), 64-87.

Danaraj (T. J.), 1956. - Proc. Alumni. Ass., Malaya, 9, 172 (in Nelson et al., 1962).

EuZEBy (J.), 1958. - Diagnostic expérimental des helminthoses animales, Vigot Frères, Editeur, Paris, $367 \mathrm{pp}$.

EUZEBY (J.), 1961. - Les maladies vermineuses des animaux domestiques et leurs incidences sur la pathologie humaine (tome I, fascicule 1). Vigot Frères, Editeur, Paris, 473 pp.

Fain (A.), Herin (V.) et Thienpont (D.), 1955. - Filarioses des Bovidés au RuandaUrundi. III. Etude Parasitologique. B) Filaires des genres Setaria et Onchocerca et microfilaires sanguines et dermiques. Ann. Soc. Belge Med. Trop., 35, (5), 553-582.

Fulleborn (F.), 1914. - Zur Technik der Mikrofilarienfürbung. Zentralbl. f. Bakt. Parasitentk. u. Infektionskr., 73, 6, 427-444.

Golvan (Y. J.), 1957. - Les principales techniques de coloration des microfilaires sanguicoles. Bull. Soc. Path. exot., 50, (1), 143-157.

HAWKING (F.), 1967. - The 24-hours periodicity of microfilariae: biological mechanisms responsible for its production and control. Proc. Roy. Soc. (B), 169, 59-76.

Heisch (R. B.), Nelson (G. S.) et Furlong (M.), 1959. - Studies in Filariasis in East Africa. I. - Filariasis on the Island of Pate, Kenya. Trans. R. Soc. trop. Med. Hyg., 53, 41-53.

ITAGAKI (S.), 1958. - La filariose chez les animaux domestiques au Japon. I. - La paralysie lombaire chez la chèvre, le mouton et le cheval. Off. Internat. Epizoot., 49 bis, 526-530.

Itagaki (S.) et Taniaguchi (M.), 1954. - Pathogénicité de $S$. digitata chez les animaux domestiques (moutons, chèvres et chevaux) et son cycle évolutif. (en japonais). Jap. Jl. San. Zoology, IV.

Kadenatsir (A. N), 1956. - La sétariose chez le mouton; biologie du parasite. (en Russe). C.R. Acad. Sci. U.R.S.S., 107, 191-192.

Langeron (M.), 1949. - Précis de microscopie. Masson et $C^{i \epsilon}$, Editeurs, Paris, 1430 pp.

Nelson (G.S.), 1962. - Observations on the Development of Setaria labiato-papillosa using New Techniques for Infecting Aedes aegypti with this Nematode. J. Helminth., 36, (3), 281-296.

Nelson (G. S.), Heisch (R. B.) et Furlong (M.), 1962. - Studies in Filariasis in East Africa. II. - Filarial infections in Man, animals and mosquitoes on the Kenya Coast. Trans. R. Soc. trop. Med. Hyg., 56, 202-217.

NoE (G.), 1903. - Studi sul ciclo evolutivo della Filaria labiato-papillosa. R. Accad. Lincei, $12,387-393$. 
Poirier (M.) et Deschiens (R.), 1951. - Sur une modalité de la technique de coloration des microfilaires. Méthode panoptique. Bull. Soc. Path. exot., 44, (11-12), 748-749.

Sноно (C.), 1959. - Sur les filaires chez les Equidés et les Bovidés. Rev. Elev. et Med. Vét., Pays Tropicaux, 12, (1), 43-52.

Shoнo (C.) et NaIR (V. K.), 1960. - Studies of cerebrospinal nematodiasis in Ceylon. VII. - Experimental production of cerebrospinal nematodiasis by the inoculation of infective larvae of Setaria digitata into susceptible goats. Ceylon Vet. J., 8, 1-11.

Williams (H.E.), 1955. - Studies on the bovine filarial Setaria cervi (Rudolphi, 1819). Parasitology, 45, (1-2), 56-62.

YEH (L. S.), 1959. - A revision of the nematode genus Setaria Viborg, 1795; its host parasite relationship, speciation and evolution. J. Helminth., 33, 1-98. 